\title{
Localização da lesão e níveis de stress em pacientes dermatológicos ${ }^{1}$
}

\author{
Site of the lesion and stress levels of \\ dermatologic patients
}

\author{
Martha Wallig Brusius LUDWIG ${ }^{2}$ \\ Margareth da Silva OLIVEIRA ${ }^{2}$ \\ Marisa Campio MÜLLER ${ }^{3}$ \\ Ângela Maria Barbosa Ferreira GONÇALVES ${ }^{4}$
}

\begin{abstract}
Resumo
O stress repercute nas doenças de pele. A pele é um órgão visível, que tem importância nas relações interpessoais e pode trazer prejuízos quando lesionada. Neste estudo, avaliou-se stress e localização da lesão dermatológica e a associação entre essas variáveis, comparando níveis de stress em pacientes com lesões no rosto e/ou mãos e pacientes com lesões em outras partes do corpo. Tratou-se de um estudo transversal, descritivo, de associação. Participaram 205 pacientes, que responderam ao Inventário de Sintomas de Stress para Adultos de Lipp, às Fichas de Dados Sócio-Demográficos e de Localização da Lesão. Quanto aos resultados, houve presença de sintomas de stress na maioria dos pacientes; a fase de resistência e a sintomatologia psicológica foram predominantes. Na comparação entre grupos com diferentes localizações de lesão, não houve diferenças significativas quanto aos resultados de stress ( $p=0,495 ; p=0,873$ e $p=0,815$ ). Os achados corroboram a necessidade de um atendimento biopsicossocial aos pacientes.
\end{abstract}

Unitermos: Dermatologia. Lesões. Psicologia. Stress.

\begin{abstract}
The skin is a visible organ, playing an important role in interpersonal relations, and when wounded may cause distress. Stress has influences on several skin diseases just as these are stress generators. This study assessed stress and dermatological lesion sites and the association between these variables, comparing stress levels in patients with lesions on the face and/or hands and patients with lesions on other parts of the body. It was a descriptive, association-based, cross-study. The sample was made of 205 patients, who filled out the Lipp's Stress Symptom Inventory for Adults, the Socio-demographical Data File and the Lesion Site File. No significant differences were found in the stress results between the groups, but the presence of stress symptoms in the majority of patients is worth highlighting, being the resistance phase and the psychological symptomatology the predominant ones. These was no significant difference in stress results between the two groups $(p=0,495 ; p=0,873$ and $p=0,815)$. These findings corroborate the need of a biopsychosocial care to the pacientes.
\end{abstract}

Uniterms: Dermatology. Lesions. Psychology. Stress.

$\operatorname{rrv}$

1 Artigo elaborado a partir da dissertação de M.W.B. LUDWIG, intitulada "O adoecimento da pele: um estudo de qualidade de vida, stress e localização da lesão dermatológica". 2007. Projeto de pesquisa desenvolvido com apoio do Conselho Nacional de Desenvolvimento Científico e Tecnológico (processo CNPa n०131848/2005-4)

2 Pontifícia Universidade Católica do Rio Grande do Sul, Programa de Pós-Graduação em Psicologia. Av. Ipiranga, 6681, prédio 11, $9^{\circ}$ andar, sala 932 , 90619-900, Porto Alegre, RS, Brasil. Correspondência para/Correspondence to: M.W.B. LUDWIG.

3 Universidade do Vale do Rio dos Sinos, Faculdade de Psicologia, Curso de Especialização em Psicossamática. Porto Alegre, RS, Brasil.

4 Sociedade Brasileira de Dermatologia e Clínica. Porto Alegre, RS, Brasil. 
A pele constitui o maior órgão do corpo humano, envolvendo-o e protegendo-o por completo. Montagu (1988, p.30) fala da pele como o espelho do funcionamento do organismo: sua cor, textura, umidade, secura, e cada um de seus demais aspectos refletem nosso estado de ser, psicológico e também fisiológico ..., é espelho de nossas paixões e emoções, sendo como uma roupagem contínua e flexível, além de ter a mesma origem embrionária que o sistema nervoso. Reveste e limita o organismo, protegendo-o de agentes externos, e é importante na manutenção do equilíbrio do meio interno (Souza et al., 2005). Por configurar o órgão de limite entre mundo interno e externo, a pele, quando lesionada, pode trazer constrangimento ao indivíduo. Nesse sentido, Strauss (1989, p.1221) refere que "ao mesmo tempo em que nos protege, é a fachada que nos expõe".

Alguns autores sugerem dificuldades quando da exposição das lesões de pele, como Fonseca e Campos (2003), ao mencionar que lesões visíveis causam constrangimento nos pacientes, e Azulay R. D. e Azulay D. R. (1992), que postulam que "convém lembrar que o indivíduo com a pele comprometida, sobretudo em áreas descobertas, dificilmente deixa de ficar envergonhado, ansioso e triste". Nadelson (1978) complementa esta idéia, referindo que a doença de pele, na mente popular, pode muitas vezes estar ligada à idéia de sujo, feio e contagioso, devendo permanecer afastado. Nesse sentido, está implicada a relação entre doenças de pele e aspectos emocionais, mais especificamente o stress, neste estudo. Frente a dados como esses, percebe-se a importância de pesquisas nesta área, explorando as repercussões dos problemas dermatológicos e buscando sensibilizar a população em geral, assim como os profissionais que trabalham com esses pacientes, para que o atendimento abarque as diferentes dimensões do ser humano.

Embora a existência de uma relação entre as alterações psicológicas e as doenças dermatológicas não seja um tema novo, ainda não é possível definir com clareza que alterações psicológicas são capazes de causar alterações dermatológicas, ou se as enfermidades cutâneas crônicas carregam, necessariamente, como qualquer outro transtorno com essas caracte344 rísticas, alterações psicopatológicas significativas
(Grimalt, Peri \& Torres, 2002). Talvez essa seja uma das razões pelas quais muitas vezes o atendimento ao paciente aconteça de forma dissociada, ou seja, o dermatologista se responsabiliza somente pela dimensão orgânica, a pele, e o psicólogo, pelos aspectos emocionais, de forma isolada.

Segundo Mingorance, Loureiro, Okino e Foss (2001), muitos estudos têm sido realizados associando o funcionamento mental do paciente com psoríase a correlatos psíquicos: o impacto emocional da doença, o aumento de preocupações e a ansiedade estão associados à piora das lesões, o alto nível de depressão, à presença de distúrbios no ambiente familiar e outros. Os temas das pesquisas revelam que a dermatose não está relacionada somente à pele, às questões orgânicas, mas que influencia e é influenciada por outros aspectos da vida do indivíduo, tanto questões emocionais quanto o próprio contexto em que vive.

De acordo com Sampaio e Rivitti (2001), é indiscutível que os fatores emocionais influenciam inúmeras dermatoses que, de outro lado, atuam no estado mental. A partir da idéia de que toda doença humana é psicossomática, pois incide em um ser provido de soma e psique, inseparáveis anatômica e funcionalmente (Mello Filho, 2002), o adoecimento (neste caso, da pele), pode repercutir em diversos âmbitos da vida do indivíduo.

Quando se pensa na inseparabilidade da psique e do corpo, ou das emoções e da pele, o stress é uma variável importante. Desde os estudos de Selye, em 1936, o stress é um fator que está relacionado ao surgimento e desenvolvimento de doenças. Vivas e Serritiello (2002) referem que extensos estudos indicam que o stress emocional pode exacerbar alguns eventos, como na psoríase, por exemplo. Steiner e Perfeito (2003) corroboram esta idéia, mencionando que "o stress físico ou emocional tem repercussões em inúmeras dermatoses e estas, indiscutivelmente, também são geradoras de stress" (p.113).

A literatura, de modo geral, enfoca a influência do stress no desenvolvimento das dermatoses (Asadi \& Usman, 2001; Picardi, Porcelli, Pasquini \& Fassoni, 2006; Taborda, Weber \& Freitas, 2005), e poucos são os estudos que avaliam o contrário, ou seja, o quanto a lesão de pele interfere no grau de stress do indivíduo. Lipp (1996) refere as doenças relacionadas ao stress e cita a psoríase entre as mais estudadas, sugerindo que "doenças relacionadas ao stress sejam classificadas como 
psicofisiológicas, termo este que enfatiza a correlação entre aspectos físicos e psicológicos que se manifestam de modo quase que inseparável durante a resposta ao stress".

Hoffmann, Zogbi, Fleck e Müller (2005) mencionam que o vitiligo está associado a fatores psicológicos, visto que, no estudo de Müller (2005), o aparecimento da doença se deu após situação de stress emocional. O'leary, Creamer, Higgins e Weinman (2004) estudaram as causas atribuídas pelos pacientes psoriáticos à sua doença, e encontraram uma grande proporção dos pacientes referindo o stress como a causa da sua doença. Esta crença está associada a um baixo bem-estar psicológico e à percepção de que a psoríase tem um impacto emocional muito grande. Apesar da prevalência desta crença, os níveis de stress, mesmo fortemente associados ao humor e à qualidade de vida, não foram associados com a severidade da psoríase.

O stress psicológico e a ansiedade têm sido reconhecidos clinicamente pelos dermatologistas como fatores relacionados à piora das lesões de pele (Fortune, Main, O'Sullivan \& Griffiths, 1997). No estudo de AmorimGaudêncio, Roustan e Sirgo (2004), que avaliou dois grupos, um com e outro sem dermatoses, foram encontradas associações entre altos níveis de ansiedade e stress em pessoas que sofrem de dermatoses inflamatórias crônicas.

Panconesi e Hautmann (1996), em um artigo sobre a psicofisiologia do stress na dermatologia, referem que os fatores genéticos e de percepção podem influenciá-lo, sendo a percepção do indivíduo sobre o desafio que o estímulo específico implica o fator mais importante.

Em relação aos problemas dermatológicos, Azambuja (2000) menciona que existem íntimas ligações entre o sistema nervoso e a pele, o que a torna extremamente sensível a emoções, de forma que qualquer problema de pele, independentemente de sua causa, tem impacto emocional. O autor discorre ainda que o stress, seja físico, psicológico ou ambiental, provoca no indivíduo reações como taquicardia, diminuição da temperatura do corpo, entre outras, e que atualmente um número crescente de cientistas tem aceitado o stress como fator precipitante de qualquer doença, não apenas das psicossomáticas.
Além disso, Azambuja (2000) refere a insustentabilidade da concepção cartesiana de mente e corpo, falando do campo da psiconeuroimunologia, que "cria um novo contexto em que não existem partes separadas, e tudo influencia tudo, tornando-se absurdo enfocar a patologia e o tratamento unicamente do corpo e, pior ainda, de uma de suas partes sem considerar o funcionamento geral" (p.407).

No que tange à localização das lesões de pele, a maior parte dos estudos avalia qualidade de vida. Schmid, Jaeger e Lamprecht (1996) mencionam que os pacientes com lesões na região do baixo ventre e genital relatam sentimentos de estigmatização com maior intensidade do que pacientes acometidos em outras áreas do corpo. Quando se discute local da lesão, está implicada a questão da aparência física. No estudo de Mingnorance, Loureiro e Okino (2002), os pacientes que relataram insatisfação quanto à aparência física, quando comparados ao grupo com percepção satisfatória da aparência, apresentaram prejuízo significativamente maior nas atividades rotineiras $(p<0,05)$ e na qualidade de vida geral $(p<0,01)$.

No estudo de Ludwig e Oliveira (2007), que avaliou qualidade de vida e localização da lesão dermatológica, não foram encontradas diferenças significativas na comparação entre dois grupos (rosto e/ou mãos; outras partes do corpo), sendo o número de associações entre os instrumentos de qualidade de vida SF-36 (qualidade de vida geral) e DLQI-BRA (qualidade de vida específica) muito superior no grupo com lesões em rosto e/ou mãos. Quando se fez a divisão mais detalhada da localização da lesão, em cinco grupos, houve diferenças significativas, sendo o grupo com a maior mediana aquele com lesões generalizadas (rosto e mãos e outras). As autoras inferem que, independentemente da locali-zação da lesão no corpo, o sentimento de exposição e os prejuízos a que fica submetido o paciente derma-tológico são semelhantes, seja a lesão mais exposta ou menos exposta ao olhar do outro.

Kadyk, McCarter e Achen (2003) estudaram qualidade de vida em pacientes com dermatite de contato, encontrando um escore significativamente pior no item relacionado à aparência da pele, em comparação aos que não têm a face acometida. Além disso, pacientes com a face afetada sentem um maior grau de prejuízo, que tende a ser significativo, e apresentam escores melhores do que aqueles sem acometimento 
da face em duas questões: medo de ser despedido e dificuldade de usar as mãos no trabalho.

Não houve diferenças significativas, nas escalas de sintomas ou emoções, entre ter ou não as mãos afetadas, no mesmo estudo. Os autores da pesquisa referem diversos relatos de que a dermatite de contato nas mãos afeta negativamente as habilidades para trabalhar e continuar as atividades diárias normais.

Também M.A. Gupta e A.K. Gupta (2003) e Hautman e Panconesi (1997) apontam a importância de considerações sobre o tema da localização da lesão, referindo que até mesmo uma doença benigna em partes do corpo "carregadas de emoção" (por exemplo, o rosto, a cabeça e o pescoço) pode debilitar particularmente o paciente.

Este estudo tratou da avaliação do stress em pacientes portadores de dermatoses com diferentes localizações da lesão dermatológica, verificando associações entre stress e localização da lesão e comparando pacientes com lesões em rosto e/ou mãos com aqueles cujas lesões se localizavam em outras partes do corpo.

\section{Método}

Desenvolveu-se um estudo transversal, descritivo, de associação, no qual foram avaliados e comparados dois grupos, de acordo com o local da lesão dermatológica: um grupo foi composto por pacientes com lesões mais expostas (rosto e/ou mãos), e o outro, por pacientes somente com lesões menos expostas (outras partes do corpo, que não rosto e/ou mãos).

\section{Participantes}

A amostra deste estudo foi por conveniência, constituída por 205 pacientes, adultos, portadores de dermatose, que estiveram em atendimento nos ambulatórios de dermatologia do Hospital São Lucas da Pontifícia Universidade Católica do Rio Grande do Sul (HSL-PUC-RS) e do Complexo Hospitalar Santa Casa de Porto Alegre, no período de fevereiro a julho de 2006. Foram excluídos da pesquisa pacientes com diagnóstico de câncer de pele ou de alguma dermatose/ doença maligna, pacientes com HIV/AIDS, que pudessem agravar os níveis de stress. Durante a pesquisa, considerou-se pertinente excluir os pacientes que apresentassem lesões ulceradas nas pernas, que, segundo D.A. Azulay e R.A. Azulay (2004), com freqüência têm origem vascular e sanguínea. Tais lesões, por serem mais incapacitantes que as demais dermatoses, inclusive do ponto de vista da locomoção, trazem maiores prejuízos ao indivíduo, podem exigir repouso e interferir nos resultados de qualidade de vida tanto geral, quanto específica. Assim, contou-se inicialmente com 214 sujeitos voluntários, dos quais nove foram excluídos.

\section{Instrumentos}

Para caracterizar a amostra quanto aos dados sócio-demográficos e à dermatose, utilizou-se ficha de dados sócio-demográficos e diagnósticos, e ficha de localização da lesão dermatológica para verificar as partes do corpo acometidas.

Foi utilizado o Inventário de Sintomas de Stress para Adultos de Lipp (ISSL) (Lipp, 2000). Esse instrumento fornece três resultados: verifica a presença ou não de sintomas de stress, o tipo de sintomatologia existente (se somática, psicológica, ou ambas) e a fase do stress em que o sujeito de encontra. O primeiro resultado permite diagnosticar se o indivíduo está ou não com stress. Questões como mãos e pés frios, aumento da sudorese, problemas com a memória, cansaço constante, insônia, entre outros, exemplificam os sintomas somáticos. Os sintomas psicológicos podem ser ilustrados pelas questões: aumento súbito de motivação, irritabilidade excessiva, sensação de incompetência em todas as áreas ou angústia/ansiedade diárias.

As fases do stress são baseadas no modelo quadrifásico. A fase de alerta é a fase positiva do stress, em que o indivíduo se prepara para a ação, tornando-se mais atento, forte e motivado. O indivíduo entra na fase de resistência quando a fase de alerta se mantém por longo período, ou quando ficam acumulados novos estressores e o organismo tenta evitar o desgaste total. Nesta fase, a vulnerabilidade da pessoa a vírus e bactérias fica acentuada. Na fase de quase-exaustão, a tensão excede o limite do gerenciável, fazendo com que a pessoa consiga pensar lucidamente, rir e trabalhar, entre 
outras tarefas, mediante grande esforço. A fase de exaustão é a mais séria, a mais negativa do stress. Nesta fase, há um desequilíbrio interior muito grande, podendo ocorrer doenças como pressão alta, psoríase e vitiligo.

\section{Procedimentos}

Esta pesquisa teve apreciação e aprovação da Comissão Científica da Faculdade de Psicologia (FAPPSI) e do Comitê de Ética em Pesquisa da Pontifícia Universidade Católica do Rio Grande do Sul, ofício n¹34/05, bem como foi aprovada sem restrições pelo Comitê de Ética em Pesquisa do Complexo Hospitalar Santa Casa de Porto Alegre (CEP/ISCMPA), parecer no 850/05. Os pacientes tiveram participação voluntária, mediante assinatura do Termo de Consentimento Livre e Esclarecido.

Realizou-se a coleta de dados de forma individual, nos próprios ambulatórios de dermatologia dos hospitais, em uma sala disponível, resguardando a privacidade do paciente. 0 mesmo era convidado a participar da pesquisa após a consulta com o dermatologista. No HSL-PUC-RS, o médico encaminhava o paciente para a pesquisa após a consulta, e no Complexo Hospitalar Santa Casa, a pesquisadora acompanhava a consulta dermatológica, convidando o paciente para a pesquisa ao final da mesma.

A análise de dados foi feita por meio de Estatística Descritiva e do Qui-Quadrado. Nas conclusões foi utilizado o nível de significância de 5\%. Os dados foram computados e analisados no programa Statistical Package for Social Sciences versão 11.5.

\section{Resultados}

Participaram desta pesquisa 205 sujeitos, com idade média de 47,43 anos (desvio-padrão 15,07); estando grande parte dos sujeitos (46,6\%) entre 40 e 49 anos e entre 20 e 29 anos (30,5\%). Quanto ao tempo de diagnóstico, 66,3\% tinha até 5 anos, e 37,7\% mais de 5 anos; 85 sujeitos não participaram desta análise, por não constar a informação no protocolo.

A maior parte da amostra era do sexo feminino (67,5\%). Em relação à escolaridade, 39,3\% possuía Ensino
Fundamental incompleto, 22,8\%, Ensino Médio completo, e os demais estavam distribuídos nas outras escolaridades, sendo a minoria (10,6\%) com Ensino Superior completo ou incompleto. A maioria dos sujeitos tinha um cônjuge ou companheiro (51,8\% destes pertenciam ao grupo com lesões em rosto e/ou mãos e 54,7\% ao grupo com lesões em outras partes do corpo). A maior parte dos pacientes trabalhava (55,1\%), estava aposentada (25,4\%) ou era"do lar"(10,2\%); os demais estavam em situação de benefício (3,4\%), estudando $(2,9 \%)$ ou desempregados (2,9\%). Quanto aos dados sócio-demográficos (idade, $p=0,878$; escolaridade, $p=0,511$ e sexo, $p=0,323)$, não houve diferença significativa entre os grupos; foi então possível a comparação pela homogeneidade dos grupos. Em relação aos diagnósticos, os mais freqüentes na amostra total foram manchas, psoríase, dermatites, fungos ou vitiligo (Tabela 1).

Os dados foram analisados a partir da distribuição da amostra total em dois grupos, conforme o local da lesão dermatológica. Dos 141 sujeitos com lesões em rosto e/ou mãos, 42 (29,8\%) apresentavam diagnóstico de vitiligo ou psoríase e 99 (70,2\%), de outras dermatoses. No grupo de 64 pacientes com lesões em outras partes do corpo, 22 (34,4\%) tinham diagnóstico de vitiligo ou psoríase e 42 (65,6\%) tinham diagnóstico de outras dermatoses. Realizou-se o Teste de QuiQuadrado entre os sujeitos com vitiligo ou psoríase e

Tabela 1. Distribuição e percentual dos diagnósticos da amostra total. Porto Alegre, 2006.

\begin{tabular}{lcr}
\hline Diagnóstico & $\mathrm{n}$ & $\%$ \\
\hline Acne & 9 & 4,36 \\
Manchas & 25 & 12,13 \\
Ceratoses $^{1}$ & 10 & 4,84 \\
Psoríase $^{2}$ & 47 & 22,80 \\
Dermatites $^{2}$ & 20 & 9,70 \\
Fungos $^{3}$ & 25 & 12,13 \\
Queda de cabelo & 9 & 4,36 \\
Vitiligo $^{\text {Outros diagnósticos menos frequentes }}{ }^{4}$ & 18 & 8,73 \\
\hline
\end{tabular}

${ }^{1}$ Ceratoses actínica, seborréica, pilar; ${ }^{2}$ dermatites ocre, atópica, crônica, de contato, herpertiforme, seborréica; ${ }^{3}$ Cândida, fungos, onocomicose; ${ }^{4}$ alopécia, artrite psoriásica, líquen plano ou esclero-atrófico, lupus discóide ou eritematoso túmido, pêlos encravados, alergia, verruga, cisto, eczema, granuloma, hanseníase, ictiose, melanose actínica, melasma, dermatofibroma, neurodermatite, paniculite, púrpura pigmentar, urticária, sardas, herpes, pele seca, escabiose, foliculite, rosácea, xantelasma. O percentual não totaliza 100\% por haver pacientes com mais de um problema de pele. 
os sujeitos com outras dermatoses, e não foram encontradas diferenças significativas entre esses dois grupos $(p=0,228)$.

Quanto aos resultados do ISSL (Tabela 2), encontrou-se que a maioria dos sujeitos apresentava sintomas de stress e encontrava-se na fase de resistência, com a sintomatologia psicológica como predominante.

Na comparação entre os dois grupos de localização da lesão dermatológica, os resultados se mantiveram tal qual a amostra total, demonstrando que a maioria dos pacientes apresentava sintomas de stress, estava na fase de resistência e tinha a sintomatologia psicológica como predominante. Tais dados demonstram que, tanto no que se refere a apresentar ou não sintomas de stress, quanto no que se refere à fase e à sintomatologia do mesmo, é indiferente a localização da lesão dermatológica, ou seja, não há evidências de associação entre os resultados do inventário e o local da lesão (Tabela 3).

\section{Discussão}

O alto índice de sintomas de stress destacou-se entre os dois grupos, e não houve evidências de associação entre os resultados do ISSL e o local da lesão. A maior parte da amostra apresentou sintomas de stress, além de grande parte estar na fase de resistência, caracterizada pela grande utilização de energia, podendo gerar sensação de desgaste generalizado sem causa aparente e dificuldades com a memória, entre outras conseqüências (Lipp, 2003).

Assim como no estudo de Silva, Müller e Bonamigo (2006), a fase de resistência em que se encontrava a maioria dos sujeitos desta pesquisa evidencia uma tentativa automática de manter a homeostase interna. Souza et al. (2005) concordam com esta idéia

Tabela 2. Freqüências e percentuais em relação ao stress. Porto Alegre, 2006.

\begin{tabular}{lcc}
\hline Apresenta sintomas de stress & $\mathrm{n}$ & $\%$ \\
\hline Sim & 135 & 65,9 \\
Não & 70 & 34,1 \\
Fase do stress & 5 & \\
Alerta & 5 & 2,4 \\
Resistência & 104 & 50,7 \\
Quase-Exaustão & 16 & 7,8 \\
Exaustão & 10 & 4,9 \\
Sem diagnóstico & 70 & 34,1 \\
Sintomatologia mais presente & & \\
Área psicológica & 96 & 46,8 \\
Área física & 21 & 10,2 \\
Sem diagnóstico & 70 & 34,1 \\
Ambas & 18 & 8,8 \\
\hline
\end{tabular}

Tabela 3. Comparação de freqüências e percentuais do Inventário de Sintomas de Stress Jpara Adultos de Lipp entre os grupos. Porto Alegre, 2006.

\begin{tabular}{|c|c|c|c|c|c|}
\hline \multirow{3}{*}{ Variável } & \multicolumn{4}{|c|}{ Grupos } & \multirow{3}{*}{$p$} \\
\hline & \multicolumn{2}{|c|}{ Rosto e/ou mãos } & \multicolumn{2}{|c|}{ Outras partes } & \\
\hline & $\mathrm{n}$ & $\%$ & $\mathrm{n}$ & $\%$ & \\
\hline \multicolumn{6}{|c|}{ Apresenta sintomas de stress } \\
\hline Sim & 95 & 67,4 & 40 & 62,5 & 0,495 \\
\hline Não & 46 & 32,6 & 24 & 37,5 & \\
\hline \multicolumn{6}{|l|}{ Fase do stress } \\
\hline Alerta & 4 & 2,8 & 1 & 1,6 & \\
\hline Resistência & 74 & 52,5 & 30 & 46,9 & \\
\hline Quase-exaustão & 11 & 7,8 & 5 & 7,8 & 0,873 \\
\hline Exaustão & 6 & 4,3 & 4 & 6,3 & \\
\hline Sem Diagnóstico & 46 & 32,6 & 4 & 37,5 & \\
\hline \multicolumn{6}{|c|}{ Sintomatologia mais presente } \\
\hline Área psicológica & 66 & 46,8 & 30 & 46,9 & \\
\hline Área física & 16 & 11,3 & 5 & 7,8 & \\
\hline Ambas & 13 & 9,2 & 5 & 7,8 & \\
\hline Sem diagnóstico & 46 & 32,6 & 24 & 37,5 & 0,815 \\
\hline
\end{tabular}

" $p$ " dado pelo teste de Qui-quadrado. 
quando falam da função da pele de revestir e delimitar o organismo, protegendo-o de agentes externos e sendo fundamental na manutenção do equilíbrio interno.

Segundo Lipp (2000) e Selye $(1956,1965)$, pode haver uma quebra da resistência se persistirem os fatores estressantes em freqüência ou intensidade, podendo o indivíduo passar para uma fase mais grave do stress. Desta forma, fica evidenciada a importância de intervenções junto a esses pacientes, com o objetivo de ajudá-los a controlar o stress, prevenindo situações mais sérias.

No aspecto da sintomatologia, entre sintomas somáticos, psicológicos ou ambos, os sujeitos apresentaram predominantemente sintomatologia psicológica, ilustrada por sintomas como angústia/ansiedade diária, dúvida quanto a si próprios, vontade de fugir de tudo, entre outros. Souza et al. (2005) referem que o stress emocional comumente acompanha os problemas dermatológicos, influenciando, então, as alterações da pele.

Taborda et al. (2005), avaliando prevalência de sofrimento psíquico em pacientes com dermatoses psicocutâneas (as presentes em seu estudo foram acne vulgar, vitiligo, psoríase, urticária, dermatite atópica e alopécia areata), apontam que as doenças crônicas, de longa duração e inestéticas, como o vitiligo, podem estar associadas a maior grau de sofrimento psíquico, dado que também corrobora os achados da pesquisa aqui relatada. As autoras ressaltam também a questão da aparência, já que "as lesões hipocrômicas do vitiligo são extremamente inestéticas, muitas vezes trazendo disfunção emocional que requer intervenção psicológica" (p.354), o que salienta o prejuízo emocional destes pacientes.

A autora do instrumento utilizado no estudo aqui descrito (Lipp, 2000) refere que uma maior incidência em determinada área demonstra a maior vulnerabilidade da pessoa na mesma, por exemplo: há pessoas que, quando estressadas, podem desenvolver ansiedade ou depressão, e outras que podem apresentar algum sintoma físico.

Esse entendimento do instrumento, bem como os resultados aqui obtidos, reforçam a noção de inseparabilidade do corpo e da psique concebida na psicossomática, para a qual não existe uma dualidade mente- corpo. Isto porque os pacientes possuíam um sintoma físico desenvolvido, a dermatose, e apresentavam uma vulnerabilidade maior para desenvolver stress na área psicológica, o que evidencia o acometimento do sujeito como um todo, bem como a constante inter-relação de aspectos físicos e emocionais.

Nesta pesquisa, os pacientes mostraram-se afetados em sua totalidade: o corpo (aspectos físicos), evidenciado pela dermatose, e o psiquismo (aspectos psicológicos), o que se pode constatar pela sintomatologia psicológica predominante, bem como pelo sofrimento relatado por essas pessoas.

Nessa linha de raciocínio, a pele parece ser a "válvula de escape" via corpo nesses pacientes; por meio dela são manifestados os sofrimentos. Desta forma, pode-se pensar no processo de stress nos pacientes dermatológicos como um excesso de sofrimento emocional que se manifesta na pele, e não estão presentes, portanto, outras sintomatologias físicas do stress (insônia, taquicardia, sudorese, entre outras), predominando a sintomatologia psicológica.

Relacionado a esse entendimento, Souza et al. (2005) apontam o acompanhamento psicoterápico focalizado nos fatores percebidos como desencadeantes da psicodermatose, bem como nos fatores de stress presentes no dia-a-dia, como uma possibilidade de encontrar "uma nova forma de simbolização em que a pele deixe de ser o veículo de expressão do sofrimento" (p.173).

A pesquisa aqui relatada averiguou a influência da lesão de pele nos níveis de stress, a fim de verificar diferenças entre indivíduos com lesões menos ou mais expostas, e não o stress como fator desencadeante da dermatose. Futuros estudos poderiam aprofundar a questão psicossomática, avaliando severidade da doença, intensidade de sintomas e local da lesão de acordo com a sintomatologia do stress, investigando se existe associação entre sintomatologia psicológica e severidade da doença, por exemplo.

Levando em consideração que o sistema nervoso e a pele têm a mesma origem embrionária (Montagu, 1988) e estão, portanto, em estreita ligação, pode-se ressaltar o quanto o stress na fase de resistência determina uma piora na dermatose, e o quanto se deveria propor trabalhos e intervenções para atingir múltiplas 
variáveis no tratamento. É preciso intervir além da superfície da pele, e buscar alcançar também o psiquismo e o comportamento.

Os resultados deste estudo permitem inferir que os prejuízos em relação a ter ou não sintomas de stress, sua fase e a sintomatologia predominantes são semeIhantes nos pacientes dermatológicos, independentemente da localização da lesão de pele. A presença de uma doença de pele provoca stress, independentemente de ser ou não exposta, e este é um dado que reforça a necessidade de um trabalho médico-psicológico integrado junto a esses pacientes (Müller, 2005).

Uma hipótese para não terem sido encontradas diferenças significativas em relação ao stress nos dois grupos de localização da lesão dermatológica pode estar relacionada à própria variável aqui estudada, o stress, pois talvez os maiores prejuízos em termos de visibilidade das lesões estejam relacionados à auto-estima e à auto-imagem, mais especificamente, visto que alguns autores referem que lesões visíveis causam constrangimento nos pacientes (Fonseca \& Campos, 2003). Além disso, a intensidade do prejuízo adaptativo varia de acordo com a percepção do paciente sobre a sua dermatose, embora se saiba do alto impacto psicossocial decorrente da psoríase, por exemplo (Root, Kent \& Al'Abadie, 1994).

Nesse sentido, deve-se levar em consideração a singularidade de cada pessoa, pois a forma de interpretar a sua doença e de lidar com a mesma depende também de aspectos muito individuais, o que é reforçado pelo estudo de Silva et al. (2006), que avaliou stress e estratégias de coping em pacientes com psoríase, buscando identificar as diferentes formas de o paciente perceber e lidar com as situações de vida. Futuros estudos poderiam avaliar as mesmas variáveis.

Uma das limitações do estudo foi não ter avaliado separadamente os sujeitos que possuíam outro problema de saúde além do de pele, o que pode ter interferido nos resultados do ISSL, por ter possivelmente captado prejuízos relativos a esta outra patologia que não a de pele.

Outros estudos sobre a pele, com o objetivo de desvendar suas repercussões na vida dos pacientes, podem ser realizados. Quanto mais claros forem os 350 fatores que influenciam a dermatose, mais instrumen- talizados ficarão os profissionais que atuam junto a esses pacientes, e mais capazes de esclarecer as variáveis e ensinar os pacientes a lidar de forma mais responsável e ativa com o seu problema de pele, melhorando tanto as condições da mesma quanto a sua vida como um todo.

\section{Considerações Finais}

Percebe-se que grande parte dos pacientes apresenta sintomas de stress, está na fase de resistência e apresenta sintomatologia predominantemente psicológica. No que tange à associação entre stress e localização da lesão, pode-se observar que ter ou não sintomas de stress, estar em uma fase mais positiva ou mais negativa do mesmo, bem como o predomínio de uma ou outra sintomatologia específica, independem da localização da lesão dermatológica.

Não foram encontradas diferenças significativas entre pacientes com diferentes locais de lesão (rosto e/ou mãos ou outras partes) quanto ao stress. Verificouse que não há evidência de associação entre os resultados do ISSL e o local da lesão, ou seja, são independentes. Tais achados sugerem que, independentemente do local da pele afetado, o stress é uma variável presente nesses pacientes, demonstrando a necessidade de um atendimento integrado médico-psicológico.

É importante salientar que, embora não existam diferenças significativas entre os dois grupos de localização da lesão neste estudo, o stress apareceu como um aspecto presente nos pacientes dermatológicos. Assim, está implicada a questão psicossomática, o entendimento do indivíduo como um ser biopsicossocial, em que os aspectos físicos e emocionais são indissociáveis, na medida em que as lesões na pele influenciam os sentimentos e as reações, sejam físicas e/ou emocionais, da pessoa acometida dermatologicamente. Também não se pode esquecer o quanto as manifestações cutâneas deixam expostas ao olhar do outro as emoções do indivíduo.

Os avanços da psicodermatologia, psiconeuroimunologia e do entendimento da influência dos aspectos emocionais nas doenças de pele caminham não apenas em direção a um entendimento integrado do indivíduo, mas principalmente em direção a um trabalho integrado médico-psicológico. 
O grande número de sujeitos na fase de resistência aponta a necessidade de os dermatologistas estarem atentos a outros fatores que contribuam para os problemas de pele, na medida em que tal fase também se caracteriza pela tentativa do organismo em restabelecer o equilíbrio interior (homeostase), quebrado na fase de alerta. Esta se caracteriza, por sua vez, pela produção e ação da adrenalina, que torna o indivíduo mais atento, forte e motivado, e é, portanto, uma fase positiva do stress (Lipp, 2000).

Por fim, os resultados desta pesquisa reforçam a necessidade de um olhar multifatorial do profissional da saúde no entendimento da doença do paciente, bem como na forma de se comunicar com o mesmo e de intervir.

Frente à complexidade que compõe o adoecimento dermatológico, o profissional da saúde que atende a esses pacientes se depara com um desafio muito grande: o de enxergar o paciente para além da sua pele, assim como auxiliá-lo para que ele próprio consiga fazer o mesmo.

\section{Referências}

Amorim-Gaudêncio, C., Roustan, G., \& Sirgo, A. (2004). Evaluation of anxiety in chronic dermatoses: differences between sexes. Revista Interamericana de Psicologia, 38 (1), 141-145.

Asadi, K., \& Usman, A. (2001). The role of psychological stress in skin disease. Journal of Cutaneous Medicine and Surgery, $5(2), 141-145$.

Azambuja, R. P. (2000). Dermatologia integrativa: a pele em um novo contexto. Anais Brasileiros de Dermatologia, 75 (4), 393-420.

Azulay, R. D., \& Azulay, D. R. (1992). Dermatologia. Rio de Janeiro: Guanabara Koogan.

Azulay, D. A., \& Azulay, R. A. (2004). Dermatologia. Rio de Janeiro: Guanabara Koogan.

Fonseca, G. F., \& Campos, L. C. (2003). Vitiligo. Acta Médica, $24,644-653$.

Fortune, D. G., Main, C. J., O'Sullivan, T. M., \& Griffiths, C. E. M. (1997). Assessing illness related stress in psoriasis: the psychometric properties of the psoriasis life stress inventory. Journal of Psychosomatic Research, 42 (5), 467-475.

Grimalt, F., Peri, J. M., \& Torres, X. (2002). Transtornos psicológicos en enfermos dermatológicos mo afecto de una psicodermatosis reconocida como tal. In F. Grimalt \&J. A. Cotterill. Dermatologyay psiquiatría: histórias clínicas comentadas (pp.37-56). España: Aula Medica Ediciones.
Gupta M. A., \& Gupta A. K. (2003). Psychiatric and psychological co-morbidity in patients with dermatologic. American Journal of Clinical Dermatology, 4 (12), 833-842.

Hautmann, G., \&Panconesi, E. (1997). Vitiligo: a psychologically influenced and influencing disease. Clinics in Dermatology, 15 (6), 879-890.

Hoffmann, F. S., Zogbi, H., Fleck, P., \& Müller, M. C. (2005). A integração mente e corpo em psicodermatologia. Psicologia: Teoria e Prática, 7 (1), 51-60.

Kadyk, D., McCarter, K., \& Achen, F. (2003). Quality of life in patients with allergic contact dermatitis. Journal of the American Academy of Dermatology, 49 (6), 1037-1048.

Lipp, M. N. (Org). (1996). Pesquisas sobre stress no Brasil. São Paulo: Papirus.

Lipp, M. N. (2000). Manual do inventário de sintomas de stress para adultos de Lipp (ISSL). São Paulo: Casa do Psicólogo.

Lipp, M. (2003). Stress: evolução conceitual. In M. N. Lipp (Org.), Mecanismos neurofisiológicos do stress: teoria e aplicações clínicas. São Paulo: Casa do Psicólogo.

Ludwig, M. W. B., \& Oliveira, M. S. (2007). O adoecimento da pele: um estudo de qualidade de vida, estresse e localização da lesão dermatológica. Dissertação de mestrado não-publicada, Pontifícia Universidade Católica do Rio Grande do Sul, Porto Alegre.

Mello Filho, J. (2002). Concepção psicossomática: visão atual. São Paulo: Casa do Psicólogo.

Mingorance, R. C., Loureiro, S. R., Okino, L., \& Foss, N. T. (2001). Pacientes com psoríase: adaptação psicossocial e características de personalidade. Medicina, 34, 315-324.

Mingnorance, R. C., Loureiro, S. R., \& Okino, L. (2002). Pacientes com psoríase: qualidade de vida e adaptação psicossocial. Anais Brasileiros de Dermatologia, 77 (2), 147-199.

Montagu, A. (1988). Tocar: o significado humano da pele. São Paulo: Summus.

Müller, M. C. (2005). Psicossomática: uma visão simbólica do vitiligo. São Paulo: Vetor Editora

Nadelson, T. (1978). A person's boudaries: a meaning of skin disease. Cutis, 21 (1), 90-3.

O'Leary, C. J., Creamer, D., Higgins, E., \& Weinman, J. (2004). Perceived stress, stress attributions and psychological distress in psoriasis. Journal-of-Psychosomatic-Research, 57 (5), 465-471.

Panconesi, E., \& Hautmann, G. (1996). Psychophysiology of stress in dermatology. Dermatologic Clinics, 14 (3), 399-422.

Picardi, A., Porcelli, P., Pasquín, P., Fassone, G., Mazzotti, E., Lega I., et al. (2006). Integration of multiple criteria for psychosomatic assessment of dermatological patients. Psychosomatics, 47 (2), 122-8.

Root, S., Kent, G., \& Al'Abadie, M. S. K. (1994). The relationship between disease severity, disability and psychological distress in patients undergoing PUVA treatment for psoriasis. Dermatology, 189 (3), 234-237.

Sampaio, S. A. P., \& Rivitti, E. A. (2001). Dermatologia. São Paulo: Artes Médicas. 
Schmid, G., Jaeger, B., \& Lamprecht, F. (1996). Dimensions of stigmatization in patients with psoriasis in a 'questionnaire on experience with skin complaints'. Dermatology, 193 (4), 304-10.

Selye, H. (1965). Stress: a tensão da vida. São Paulo: IBRASA. (Originalmente publicado em 1956).

Silva, J. D. T., Müller, M. C., \& Bonamigo, R. R. Estratégias de coping e níveis de stress em pacientes portadores de psoríase. Anais Brasileiros de Dermatologia, 81 (2), 2006.

Souza, A. P. F. S., Carvalho, F. T., Rocha, K. B., Lages, M. N., Calvetti, P. Ü., \& Castoldi, L. (2005). Associação de eventos estressores ao surgimento ou agravamento de vitiligo e psoríase. Psico, 36 (2), 167-174.

Strauss, G. (1989). Skin Disorders. Baltimore: Williams Wilkins.
Steiner, D., \& Perfeito, F. L. (2003). A relação entre stress e doenças dermatológicas. In M. E. N Lipp (Org.), mecanismos neurofisiológicos do stress: teoria e aplicações clínicas (pp.111-114). São Paulo: Casa do Psicólogo.

Taborda, M. L. V. V., Weber, M. B., \& Freitas, E. S. (2005). Avaliação da prevalência de sofrimento psíquico em pacientes com dermatoses do espectro psicocutâneo. Anais Brasileiros de Dermatologia, 80 (4), 351-4.

Vivas, C. J. R., \& Serritiello, L. S. (2002). Cinco casos demostrativos de un estudio psicomástico en enfermos psoriáticos. Archivos del Hospital Vargas, 44 (1/2), 60-67.

Recebido em: 31/1/2007

Versão final reapresentada em: 28/6/2007

Aprovado em: 26/7/2007 